\title{
Pattern of Keratitis: A Study of 500 Cases in Tertiary Hospital of Bangladesh
}

\author{
Rebeka Sultana, Md. Shahnewaz Parvez, Md. Ashraful Haque, Md. Abdul Quader, \\ and Mujtahid Mohammad Hossain
}

\section{ABSTRACT}

Background: Keratitis is a potentially serious corneal infection and a major cause of visual impairment worldwide. Most common form of which is infectious variety; pathogens may be bacteria, viruses, fungi and parasites which varies with age and predisposing factors. Topical antimicrobials and cycloplegic agents are the mainstay of treatment. Pattern of causative organisms and drug sensitivity changes from time to time and differs from one to another geographic location.

Objective: To assess and evaluate the pattern of diagnosed patient of keratitis presented in a tertiary hospital.

Material and methods: This cross-sectional study was conducted on 500 diagnosed patients of keratitis in the department of cornea of National Institute of Ophthalmology \& Hospital, a well-known tertiary care hospital with 500 beds for a period of 6 months. Patients were selected based on specific selection criteria. Detail history was taken, and thorough ocular examination was done in every patient. Corneal scrapings were taken for Gram's stain and $10 \%$ potassium hydroxide wet mount preparation. All the relevant data was recorded in a predesigned data collection sheet.

Results: The mean age of the study subjects was 38.49 years. Male $(43.2 \%)$ and female $(56.8 \%)$ ratio was almost equal. The prevalence was highest among lower economic class $\mathbf{( 5 6 . 8 \%}$ ) as usual, and lowest among affluent class $(3.8 \%)$ but not sparing them. In terms of regional distribution, majority $\mathbf{4 2 . 6 \% )}$ of the patients came from rural area, whereas $37 \%$ from urban nonslum area and $20.8 \%$ from urban slum area. The problem was mostly observed among day-laborers $(\mathbf{2 4 . 8 \%})$ with gradually declining trend among students $(20.6 \%)$, housewives $(20 \%)$, service holders $(14.2 \%)$, businessmen $(3.2 \%)$ and unemployed $(2.2 \%)$, surprisingly! Presentation of the cases were mostly in between $2^{\text {nd }}-3^{\text {rd }}$ week $(55 \%)$, while $30 \%$ patients were presented within $1^{\text {st }}$ week \& $15 \%$ after 4 weeks of diagnosis. Among the cases, it was found that $28.8 \%$ patients had corneal injury, which were caused by vegetative trauma in $16 \%$ cases, $4 \%$ dust or sand trauma, $2.8 \%$ animal injury, $2.2 \%$ stick injury, $1 \%$ nail injury and $2.8 \%$ patients had history of other injury like stone, wooden materials, flying insects, dirty wire etc. Ocular diseases predispose to corneal ulcer were present in $9 \%$ patients. These were chronic dacriocistitis, entropion and trichiasis. $1.2 \%$ patients were diabetic, and $1 \%$ patients had history of inadvertent use of steroids. On the contrary, there were no specific predisposing factors in $71.2 \%$ cases. Among the cases, 96.6\% experienced photophobia, 90\% had foreign body sensation, $\mathbf{8 8 . 4 \%}$ had reduced vision, $\mathbf{8 5 . 4 \%}$ had eye pain, $\mathbf{7 8 . 8 \%}$ suffered from watering, $\mathbf{7 3 . 6 \%}$ had discharge, $\mathbf{4 8 . 8 \%}$ had burning sensation, $12.2 \%$ had decreased corneal sensation and $\mathbf{1 1 . 2 \%}$ patients noticed white spot in cornea in first stage. Slit lamp bio-microscopic examination of the patients shows $90 \%$ patients had conjunctival injection, $70 \%$ had epithelial defect, $28.6 \%$ patients had stromal infiltration, $18.4 \%$ patients had hypopyon, $14 \%$ patients had feathery pattern, $8.8 \%$ patients had suppuration, anterior chamber reaction was present in $4.4 \%$ cases and $2.2 \%$ patients had satellite lesion. The evaluation of etiology on the basis of clinical appearance reveals $61.4 \%$ of cases were presented with viral keratitis, $23.0 \%$ fungal corneal ulcer, $11.4 \%$ with bacterial corneal ulcer, $2.2 \%$ with marginal keratitis, $0.8 \%$ with exposure keratitis, $0.8 \%$ with filamentary keratitis and $0.4 \%$ cases with Acanthamoebic keratitis at the time of presentation. While assessing the depth of keratitis of the study subjects, $73.2 \%$ found superficial while $26.8 \%$ found deep. Assessment of area of corneal involvement in terms of punctate epithelial defect showed that in $46.8 \%$ cases less than $3 \mathrm{~mm}$ of cornea was involved, 3 to $6 \mathrm{~mm}$ of the cornea involved in $19.6 \%$ cases and in $11.8 \%$ corneal involvement was found in more than $6 \mathrm{~mm}$ area. Corneal scraping done in $118(23.6 \%)$ out of 500 patients. Among them, Fungi were found in
Submitted : February 28, 2021

Published : June 09, 2021

ISSN: $2593-8339$

DOI: $10.24018 /$ ejmed.2021.3.2.746

Dr. Rebeka Sultana

Ophthalmologist,

National Institute of Ophthalmology \& Hospital, Dhaka, Bangladesh.

Dr. Md. Shahnewaz Parvez

Deputy Program Manager,

Noncommunicable Disease Control,

Dhaka, Bangladesh.

Dr. Md. Ashraful Haque*

Assistant Professor (Transfusion Medicine), Sheikh Hasina National Institute of Burn \& Plastic Surgery, Dhaka, Bangladesh

(e-mail: ashraf.djmc03@gmail.com) Professor Md. Abdul Quader

National Institute of Ophthalmology \& Hospital, Dhaka, Bangladesh

Dr. Mujtahid Mohammad Hossain National Institute of Ophthalmology \& Hospital, Dhaka, Bangladesh

(e-mail: dr.ms.parvez ${ }^{@}$ gmail.com)

*Corresponding Author 
$11.2 \%$ cases, mixed organism in $4 \%$, Bacteria in $5.6 \%$ and Acanthamoeba found in $0.4 \%$ cases while $2.4 \%$ remains unidentified.

Conclusion: Analysis of the study findings shows diversity in the pattern, presentation, predisposing factors of different form of keratitis which may be helpful as basis of further study on this problem.

Keywords: Keratitis, Pattern, NIOH, Corneal, Scraping, Vegetative, Ocular, Photophobia, Filamentary, Acanthamoeba, Ulcer, Hypopyon, Satellite, Fungal, Marginal, Stromal, Slit Lamp.

\section{BACKGROUND}

The cornea, the clear part of the eye through which light passes, is subject to many infections and to injury from exposure to foreign objects. Infection and injury cause inflammation of the cornea and is called keratitis. Both infectious and immune mechanisms are important in the development of this sight threatening condition [1], [2]. Keratitis can progress rapidly with corneal destruction through pathological wound healing within 24-48 hours [3][5]. Any organism can invade the corneal stroma when protective mechanisms are compromised. Corneal ulcers may be classified according to anatomical position and their pathogenesis [6]. Anatomical corneal ulcers can either be peripheral or central. It is usually thought that most peripheral ulcers are non-infective in nature while central ones are infective [6]. Etiologically keratitis can be classified as either infectious or non-infectious. The infectious group includes bacterial, fungal, viral and parasitic while the noninfectious group includes Mooren's, marginal, phlyctenular, traumatic and nutritional. Bacterial keratitis usually develops when the ocular defenses have been compromised. However, some bacteria are able to penetrate a normal corneal epithelium. The most common agents for bacterial keratitis are Staphylococcus aureus, Streptococcus pneumoniae and gram-negative bacilli, especially p. aeruginosa [4]. Fungal keratitis is a fatal condition which needs early diagnosis and treatment to save the patient's eye. The two main types of fungi causing keratitis are Yeast and filamentous fungi. Fungal ulcers are typically seen after injury with vegetative matter such as a thorn or wooden stick and are characterized by a relatively indolent course. Superficial keratitis may result from a number of infections, most of which are viral. Of these, the most common are herpes simplex and adenoviruses. Superficial punctuate epithelial keratitis is characterized by granular, opalescent, swollen epithelial cells with focal intraepithelial infiltrates. Numular keratitis is characterized by fine granular sub-epithelial deposits surrounded by a halo of stromal haze. Dendritic or geographic keratitis is associated with active Herpes virus replication. Disciform keratitis may be an active HSV infection of kerato-uveitis or endothelium or a hypersensitivity reaction to viral antigen in the cornea. It is less common in VZV infection. Interstitial keratitis or stromal keratitis is an inflammation of the corneal stroma without primary involvement of the epithelium or endothelium. Common causes are Syphilis, herpetic keratitis, other viral infections, tuberculosis, sarcoidosis, Cogan syndrome. Marginal keratitis is probably caused by a hypersensitivity reaction against staphylococcal exotoxins and cell wall proteins with deposition of antigen-antibody complexes in the peripheral cornea with a secondary lymphocytic infiltration. Filamentary keratopathy is a common condition that can cause discomfort. In these cases, it is thought that a loose area of epithelium acts as a focus for deposition of mucous and cellular debris. Causes are aqueous deficiency, excessive contact lens wear, corneal epithelial instability, superior limbic kerato-conjunctivitis, bullous keratopathy, neurotrophic keratopathy. Exposure keratopathy is a result of incomplete lid closure causes drying of the cornea despite normal tear production. Common causes of incomplete lid closure are facial nerve palsy, coma or parkinsonism, eczema, solar keratosis, xeroderma pigmentosum, severe proptosis, severe enopthalmos.

\section{MATERIALS AND METHODS}

A hospital based, prospective, cross sectional study was conducted for a period of 6 months from 1 July 2018 to 31 December 2018 at corneal clinic of National Institute of Ophthalmology and Hospital, Dhaka, Bangladesh. Ethical approval was obtained from ethical review committee of NIOH. Informed written consent was obtained from patient or appropriate guardian. Through a non-randomized purposive sampling, a total of 500 diagnosed cases of keratitis were studied. Demographic data, detailed history of predisposing factors, previous treatment and duration of symptoms was noted by structured questionnaire. Presenting visual acuity was recorded by Snellen's chart at 6 (six) meter distance. Pattern of keratitis including size, depth, and location of ulcer along with examination of margins, floor and infiltrations were done by using slit lamp bio microscope. Presence or absence of hypopyon, ocular adnexa (eyelids, eyelashes, conjunctiva, and lacrimal sac area) was examined by naked eye examination. Patency of the lacrimal system was assessed by performing syringing in case of nonhealing cases. Corneal surface scrapping was performed gently in every subject of diagnosed corneal ulcer. For corneal scrapping, informed verbal consent was obtained after explaining the procedure. The base and leading edges were scrapped from periphery to center. The material was smeared on two slides, one for $10 \%$ potassium hydroxide $(\mathrm{KOH})$ wet mount preparation and another for Gram's stain. Collected data were reviewed, cleaned, and finally analyzed by SPSS 17 for Windows. 


\section{RESULTS}

During this six-month study period, a total of 500 patients of keratitis attending in outpatient department of cornea were enrolled and went through the questionnaire and clinical examination and face to face interview. Results displayed in appropriate tables and graphs.

\begin{tabular}{ccc}
\multicolumn{3}{c}{ TABLE I: AGE DISTRIBUTION OF THE 500 STUDY SUBJECTS } \\
\hline Age group & Number & Percentage $(\%)$ \\
\hline 0-15 years & 24 & 4.8 \\
16-30 years & 190 & 38.0 \\
31-45 years & 134 & 26.8 \\
46-60 years & 92 & 18.4 \\
>60 years & 60 & 12.0 \\
Total & 500 & $100 \%$ \\
\hline
\end{tabular}

\begin{tabular}{|c|c|c|}
\hline Gender & Number & Percentage (\%) \\
\hline Female & 216 & $43.2 \%$ \\
\hline Male & 284 & $56.8 \%$ \\
\hline
\end{tabular}

\begin{tabular}{|c|c|c|}
\hline \multicolumn{3}{|c|}{ TABLE III: DISTRIBUTION OF SOCIOECONOMIC STATUS } \\
\hline Socio-economic Class & Cases & Percentage \\
\hline Upper class & 19 & 3.8 \\
\hline Upper middle class & 159 & 31.8 \\
\hline Lower middle class & 218 & 43.6 \\
\hline Lower class & 104 & 20.8 \\
\hline \multicolumn{3}{|c|}{ TABLE IV: DISTRIBUTION OF OCCUPATION } \\
\hline Occupation & Cases & Percentage $(\%)$ \\
\hline Day Labor & 124 & 24.8 \\
\hline Student & 103 & 20.6 \\
\hline Housewife & 100 & 20.0 \\
\hline Farmer & 75 & 15.0 \\
\hline Service holder & 71 & 14.2 \\
\hline Businessman & 16 & 3.2 \\
\hline Unemployed & 11 & 2.2 \\
\hline
\end{tabular}

TABLE V: DistribUtion OF THE PREDISPOSING FACTORS OF STUDY

\begin{tabular}{ccc} 
& SUBJECTS & \\
\hline Predisposing factors & Number & Percentage (\%) \\
\hline Trauma & 144 & 28.8 \\
a. Vegetative trauma & 80 & 16 \\
b. Mud, dust, sand & 20 & 4 \\
c. Animal matters & 14 & 2.8 \\
d. Stick & 11 & 2.2 \\
e. Nail & 5 & 1 \\
f. Others & 14 & 2.8 \\
Co-existing ocular disorder & 51 & 10.2 \\
Inadvertent use of steroid & 5 & 1 \\
No specific history of trauma & 365 & 71.2 \\
\hline
\end{tabular}

In terms of predisposing factor, majority of cases $(71.2 \%)$ had no specific history of any trauma. Only in $28.8 \%$ cases specific history of trauma was found, of which $16 \%$ were vegetative trauma, and others were from mud, dust, sand, animal matter, stick or nail etc. In $10.2 \%$ cases some coexisting ocular diseases were found (e.g., chronic dacrocystitis, entropion, trichiasis etc.). Inadvertent steroid use pertained only for $1 \%$ cases.

\begin{tabular}{ccc} 
TABLE VI: DISTRIBUTION OF THE SYMPTOMS OF STUDY SUBJECTS \\
\hline Characteristic & Number & Percentage $(\%)$ \\
\hline Photophobia & 483 & 96.6 \\
Foreign body sensation & 450 & 90 \\
Reduced vision & 442 & 88.4 \\
Eye pain & 426 & 85.4 \\
Watering & 394 & 78.8 \\
Discharge & 368 & 73.6 \\
Burning sensation & 244 & 48.8 \\
Diminished corneal & 61 & 12.2 \\
sensation & 56 & 11.2 \\
White spot &
\end{tabular}

TABLE VII: DISTRIBUTION OF SIGNS ON SLIT-LAMP EXAMINATION

\begin{tabular}{ccc}
\hline Signs & Number & Percentage $(\%)$ \\
\hline Conjunctival & 450 & 90 \\
injection & 350 & 70 \\
Epithelial defect & 143 & 28.6 \\
Stromal infiltration & 92 & 18.4 \\
Hypopyon & 70 & 14.0 \\
Feathery pattern & 44 & 8.8 \\
Suppuration & 22 & 4.4 \\
Anterior chamber & 11 & 2.2 \\
reaction & & \\
Satellite lesion & &
\end{tabular}

TABLE VIII: ETIOLOGY OF KERATITIS ON THE BASIS OF CLINICAL

\begin{tabular}{ccc}
\multicolumn{3}{c}{ APPEARANCE } \\
\hline Name of Keratitis & Number & (\%)Percentage \\
\hline Viral Keratitis & 307 & 61.4 \\
Fungal corneal ulcer & 115 & 23.0 \\
Bacterial corneal ulcer & 57 & 11.4 \\
Marginal Keratitis & 11 & 2.2 \\
Exposure Keratitis & 4 & 0.8 \\
Filamentary Keratitis & 4 & 0.8 \\
Acanthamoeba & 2 & 0.4 \\
\hline
\end{tabular}

TABLE IX: DISTRIBUTION OF DEPTH OF KERATITIS ON SLIT LAMP

\begin{tabular}{ccc}
\multicolumn{3}{c}{ EXAMINATION } \\
\hline Depth of Keratitis & Number & Percentage $(\%)$ \\
\hline Superficial & 366 & 73.2 \\
Deep & 134 & 26.8 \\
\hline
\end{tabular}

TABLE X: TYPES OF KERATITIS ON THE BASIS OF LOCATION IN THE

\begin{tabular}{ccc}
\multicolumn{3}{c}{ CORNEA } \\
\hline Location of lcer & Number & Percentage $(\%)$ \\
\hline Central & 384 & 76.8 \\
Diffuse & 101 & 20.2 \\
Peripheral & 15 & 3.0 \\
\hline
\end{tabular}

TABLE XI: INTERPRETATION OF CORNEAL SCRAPPING

\begin{tabular}{|c|c|c|}
\hline $\begin{array}{l}\text { Organism found from Corneal } \\
\text { Scrapping }\end{array}$ & Cases & Percentage $(\%)$ \\
\hline Not done & 382 & 76.4 \\
\hline Done & 118 & 23.6 \\
\hline a. Fungus Present & 56 & 11.2 \\
\hline b. Mixed & 20 & 4 \\
\hline c. Bacteria & 28 & 5.6 \\
\hline d. Not found & 12 & 2.4 \\
\hline e. Acanthamoeba & 2 & 0.4 \\
\hline
\end{tabular}

\section{DISCUSSION}

Keratitis is a major global cause of visual impairment and blindness. Most of the cases are infectious in nature and commonly affects the peoples of low socio-economic class. Infectious keratitis is a serious condition that could result in corneal scar, corneal perforation, and even blindness. Microbial keratitis is a common, potentially sight threatening ocular infection that may be caused by bacteria, fungi, or viruses. However, predisposing factors such as trauma, contact lens wear, dry eye, glaucoma, epithelial defect, systemic disease, and immunosuppressant may alter the defense mechanisms of the outer eye and permit bacteria to invade the cornea. The variables studied included demographic data, medical history, risk factors (history of ocular trauma, use of contact lenses, associated eye diseases, systemic diseases, previous ocular surgery), clinical presentation, initial and final visual acuity, medication used.

In this study, the mean age of the study subjects was $38.49 \pm 18.59$ (SD) years. Laltanpuia et al. [7], found in their study within 2012-2014 that keratitis occurred most frequently $60(78.9 \%)$ in the age group $21-65$ years. In this 
study, it was also found that people aged 16-60 years are more affected than people aged less than 15 years and above 60 years. As this age group (16-60 years) has more outdoor activities, they suffer more from ocular injury commonly. All the study findings show that most of the affected person of keratitis belongs to working group of peoples are affected by keratitis due to ocular injury, which have impact on our socio-economic status.

In his study, among 500 participants 216 (43.2\%) were female and $284(56.8 \%)$ were male. This study shows almost equal number of affected person in both genders. Possibly due to that female also involved house hold works as well as trends of female service holders increasing in Bangladesh. These findings also supported by several other studies in the different parts of the world. Laltanpuia et al [7], found almost similar result, where calculated odds are 1.37 times in males.

In the current study, among 500 patients 19 (3.8\%) patients belong to upper class group, $159(31.8 \%)$ patients belong to upper middle class group, 218 (43.6\%) patients belong to lower middle class group, and 104 (20.8\%) patients belong to lower class group.

In this study, among 500 patients $124(24.8 \%)$ were day labor, $103(20.6 \%)$ were student, 100 (20\%) were housewives, $75(15 \%)$ were farmer, $71(14.2 \%)$ were service holder, $16(3.2 \%)$ were businessman and $11(2.2 \%)$ were unemployed. Laltanpuia et al [7] found almost same result, (53:69.7) were agricultural workers/daily wage earners $(27: 35.5 \%)$ or homemakers/housewives $(26: 34.2 \%)$. In our study housewives are more affected because they were also involved more in agricultural activities.

In this study, $144(28.8 \%)$ patients had corneal injury, among them $16 \%$ patients had vegetative trauma, $4 \%$ patients had dust, sand trauma, $2.8 \%$ patients had animal injury, $2.2 \%$ patients had stick injury, $1 \%$ patients had nail injury, $2.8 \%$ patients had history of other injury like stone, wooden materials, flying insects, dirty wire etc. Ocular diseases predispose to corneal ulcer, such as chronic dacrocystitis, entropion and trichiasis were present in $10.2 \%$ patients. History of inadvertent use of steroids found in $1 \%$ patient. There were no specific predisposing factors in $71.2 \%$ of patients. Kaliamurthy, et al [8] also found the main cause as injury with vegetative matter which is $337(31.6 \%)$. As an agricultural country, this is quite natural to be affected by vegetative matter. Not only male, females are also involved in agriculture dependent works in rural areas. This may be the reason behind high incidence of agricultural trauma, as well as high incidence in rural areas.

In this study, among 500 patients $96.6 \%$ had photophobia, $90 \%$ patients had foreign body sensation $88.4 \%$ had reduced vision, $85.4 \%$ had eye pain, $78.8 \%$ suffered from watering, $73.6 \%$ of them had discharge, $48.8 \%$ of them had burning sensation, $12.2 \%$ patients suffered from decreased corneal sensation and $11.2 \%$ patients noticed white spot in cornea first.

In the present study, slit lamp bio-microscopic examination of the patients shows, $450(90 \%)$ patients had conjunctival injection, $350(70 \%)$ patients had epithelial defect, $143(28.6 \%)$ patients had stromal infiltration, 92 (18.4\%) patients had hypopyon, $70(14 \%)$ patients had feathery pattern, $44(8.8 \%)$ patients had suppuration, anterior chamber reaction was present in $22(4.4 \%)$ patients and 11 (2.2\%) patients had satellite lesion.

The above mentioned symptoms and signs are established clinical features of keratitis, although severity and intensity varies in different environmental and socio-demographic perspective and also causative organisms.

In the current study, the evaluation of etiology on the basis of clinical appearance. $307(61.4 \%)$ patients were presented with viral keratitis, $115(23.0 \%)$ presented with fungal corneal ulcer, $57(11.4 \%)$ patients presented with bacterial corneal ulcer, $11(2.2 \%)$ patients had marginal keratitis, 4 $(0.8 \%)$ patients had exposure keratitis, $4(0.8 \%)$ patients had filamentary keratitis and $2(0.4 \%)$ patients had acanthamoebic keratitis at the time of presentation (which was diagnosed elsewhere). Most common causative factors for keratitis are similar to this study finding. Incidence of the causative agents differs in different season. Age group and residing area also contributes the variation of causative agents.

In this study, assessment of the depth of keratitis of study subjects shows it was superficial up to anterior stroma 366 $(73.2 \%)$ patients and deep up to deep stroma in 134 (26.8\%) patients. According to this study finding most of the cases were diagnosed as viral keratitis and it usually spreads up to superficial stroma. This factor supports the predominant finding of superficial keratitis in this study.

In this study, assessment of area of corneal involvement shows, 234 patients had punctate epithelial defect, less than $3 \mathrm{~mm}$ of the cornea involved in 109 patients, 3 to $6 \mathrm{~mm}$ of the cornea involved in 98 patients and 59 patients had corneal involvement more than $6 \mathrm{~mm}$. As mentioned earlier that most of the cases in this study was viral keratitis and punctate epithelial defect is the earliest form of viral keratitis, so the cases of punctate epithelial defect are more in this study.

In the present study, pure fungal infection was noted in 56 $(11.2 \%)$ subjects and fungal infection associated with bacterial infection in $20(4 \%)$ subjects, bacteria found in 28 $(5.6 \%)$ patients, acanthamoeba found in $2(0.4 \%)$ patients and no organism found in $12(2.4 \%)$ patients. Puri LR et al. [9], also found the high incidence of fungal infection and low incidence of bacterial infection in their study. One reason could be due to large populations are belonging to agricultural background and rural community. The sensitivity and specificity of bacterial detection in Gram's stain is inferior to that of detecting fungal filaments by $10 \%$ $\mathrm{KOH}$ preparation. [10] [11].

Based on the above mentioned clinical patterns newer strategies may be adopted to reduce the morbidity associated with this condition are likely going to have to be multidimensional, involving corneal ulcer prevention, improved early and accurate diagnostics techniques such as next-generation sequencing, and novel antimicrobial agents to address the development of drug resistance. Adjuvant therapies that focus on modifying the immune response to the infection, thereby reducing the corneal melting and scarring that ultimately lead to poor vision, may have the greatest potential to improve clinical outcomes 


\section{CONCLUSION}

By this study, it can be concluded that microbial keratitis is more in middle aged people with low socioeconomic status working in outdoor occupation. Trauma is most common predisposing factor responsible for microbial keratitis. Microbial keratitis is most common cause of corneal blindness and the knowledge of demographic factor, predisposing factor, and microbiological pattern of microorganism responsible for microbial keratitis is very important for proper diagnosis and management of microbial keratitis.

\section{LIMITATION}

In this study, culture and sensitivity test could not be done due to insufficient budget and institutional capacity.

A multicenter cohort or case control study should be conducted including primary, secondary, and tertiary eye care center.

\section{REFERENCES}

[1] M. K. a. G. B. S. K. P. K. Shukla, "Mycotic keratitis: an overview of diagnosis and therapy," Mycoses, vol. 51, no. 3, pp. 183-274, May 2008.

[2] E. K. N. T. e. a. Keay L, "Microbial keratitis predisposing factors and morbidity," vol. 113, no. 1, pp. 109-116, 2006.

[3] T. J. Liesegang, "Contact lens-related microbial keratitis: Part I: Epidemiology," vol. 16, no. 2, pp. 125-131, 1997.

[4] M. S. \&. M. P. U. John P. Whitcher, "Corneal blindness: a global perspective," World Health Organization, 2001. [Online]. Available: https://www.who.int/bulletin/archives/79(3)214.pdf. [Accessed 26 October 2020].

[5] E. H. D. S. P. F. D. L. D. S. E. W. H. K. M. K. S. G. D S C Lam, "Incidence and risk factors for microbial keratitis in Hong Kong: comparison with Europe and North America," vol. 16, pp. 608-618, 2002.

[6] M. T. Y. K. K. K. G. P. D. S. Parul Singh, "Ocular chemical injuries and their management," Oman Journal of Ophtlalmology, vol. 6 , no. 2, pp. 83-86, 2013.

[7] P. S. U. Chhangte L, "Epidemiological and Microbiological Profile of Infectious," International Journal of Scientific and Research Publications, vol. 5, no. 2, February 2015.

[8] K. C. P. P. J. C. T. P. Kaliamurthy J, "Spectrum of bacterial keratitis at a tertiary eye care centre in India," BioMed Research International, vol. Volume 2013, 26 August 2013.

[9] S. G. Puri LR, "Microbial keratitis: A five years retrospective clinical study in tertiary eye hospital of eastern region of Nepal," Journal of Kathmandu Medical College, vol. 4, no. 14, pp. 118125, Oct-Dec 2015.

[10] S. S. Asbell P, "Ulcerative keratitis. Survey of 30 years' laboratory experience," JAMA Ophthalmology, vol. 100, no. 1, pp. 77-80, 1982.

[11] K. H. A. D. Wahi JC, "Infectious keratitis in Baltimore," Ann Ophthalmol, vol. 23, no. 6, pp. 234-237, 1991. 